\title{
Cystinosin-LKG rescues cystine accumulation and decreases apoptosis rate in cystinotic proximal tubular epithelial cells
}

\author{
Anna Taranta', Francesco Bellomo', Stefania Petrini², Elena Polishchuk³ , Ester De Leo', Laura Rita Rega', Anna Pastore', \\ Roman Polishchuk ${ }^{3}$, Maria Antonietta De Matteis ${ }^{3}$ and Francesco Emma ${ }^{1}$
}

BACKGROUND: Nephropathic cystinosis is a lysosomal storage disease that is caused by mutations in the CTNS gene encoding a cystine/proton symporter cystinosin and an isoform cystinosin-LKG which is generated by an alternative splicing of exon 12. We have investigated the physiological role of the cystinosin-LKG that is widely expressed in epithelial tissues. METHODS: We have analyzed the intracellular localization and the function of the cystinosin-LKG conjugated with DsRed (cystinosin-LKG-RFP) in Madin-Darby canine kidney cells (MDCK II) and in proximal tubular epithelial cells carrying a deletion of the CTNS gene (cystinotic PTEC), respectively.

RESULTS: Cystinosin-LKG-RFP colocalized with markers of lysosomes, late endosomes and was also expressed on the apical surface of polarized MDCK II cells. Moreover, immuneelectron microscopy images of MDCK II cells overexpressing cystinosin-LKG-RFP showed stacked lamellar membranes inside perinuclear lysosomal structures. To study the role of LKG-isoform, we have investigated cystine accumulation and apoptosis that have been described in cystinotic cells. Cystinosin-LKG decreased cystine levels by approximately 10-fold similarly to cystinosin-RFP. The levels of TNFa- and actinomycin D-inducted apoptosis dropped in cystinotic cells expressing LKG-isoform. This effect was also similar to the main isoform.

CONCLUSION: Our results suggest that cystinosin-LKG and cystinosin move similar functional activities in cells.

N ephropathic cystinosis (NC) is a rare lysosomal storage disease. It is caused by defective activity of cystinosin, a proton/cystine symporter that is ubiquitously expressed on lysosomal membranes, which leads to cystine accumulation into lysosomes and crystal formations in many tissues (1). One of the first symptoms of $\mathrm{NC}$ is renal Fanconi syndrome, secondary to a global dysfunction of proximal tubular cells. During their lifetimes, patients with NC develop several others symptoms in particular, if they are not well treated with cysteamine $(2,3)$.
Recent works have demonstrated an involvement of cystinosin in lysosomal kinetics and exocytosis (4), vesicle trafficking (4,5), autophagy, (6) and in sensing cell oxidative state (7). Studies using a cystinotic mouse model have shown decreased expression of the multi-ligand receptors megalin and cubilin in proximal tubules; this was associated with cell dedifferentiation and apoptosis $(5,8)$. Also, enhanced cell death due to activation of proapoptotic signals probably occurs in other tissues (9-11).

Cystinosin (accession number: NP_004928) is composed of seven transmembrane domains that bear two lysosomal targeting motifs (GYDQL and YFPQA) and seven N-glycosylation sites in the amino-terminal region (12). The aceview database reports various alternative splicing transcripts for the CTNS gene that encodes the cystinosin (http://www.ncbi.nlm.nih. gov/IEB/Research/Acembly). In 2008, we have identified a 400 amino acid cystinosin isoform that derives from alternative splicing of CTNS gene exon 12 (accession number: NP_001026851). The protein is identical to cystinosin, with the exception of the carboxyl-terminal tail, which is characterized by an amino acid sequence where the "GYDQL" lysosomal targeting motif is replaced by a longer tail of 37 amino acids. The expression of this isoform, which is termed cystinosin-LKG based on its last three amino acids, is not confined to the lysosome (13).

As previously demonstrated, cystinosin-LKG conjugated with a fluorescent protein and overexpressed in human epithelial cells is also expressed in the plasma membrane, where it mediates cystine influx (13). Redistribution of the protein to the plasma membrane has been shown after deleting the GYDQL and YFPQA lysosome-targeting motifs of the most abundant cystinosin isoform (12). Deleted-GYDQL cystinosin in HeLa and MDCK cells allowed L-cystine influx from the extracellular medium in the presence of proton gradient (14).

A recent immunohistochemistry analysis of the distribution of cystinosin-LKG in human tissues has revealed strong staining in epithelia, particularly in cells with intensive secretory and trafficking activities, including renal tubular cells,

'Department of Nephrology and Urology, Division of Nephrology, Bambino Gesù Children's Hospital and Research Institute, Rome, Italy; ${ }^{2}$ Confocal Microscopy core facility, Research Center, Bambino Gesù Children's Hospital, IRCCS, Rome, Italy; ${ }^{3}$ Telethon Institute of Genetics and Medicine, Naples, Italy; ${ }^{4}$ Laboratory of Metabolomics and Proteomics, Bambino Gesù Children's Hospital and Research Institute, Rome, Italy. Correspondence: Anna Taranta (anna.taranta@opbg.net)

Received 19 February 2016; accepted 10 August 2016; advance online publication 19 October 2016. doi:10.1038/pr.2016.184 
a
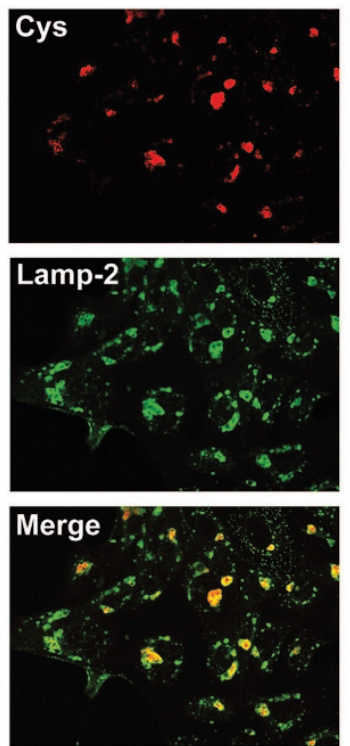

b

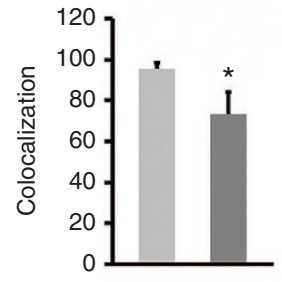

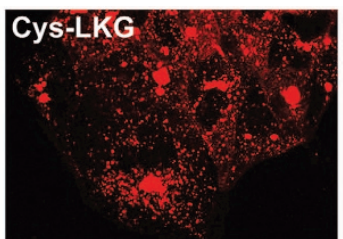
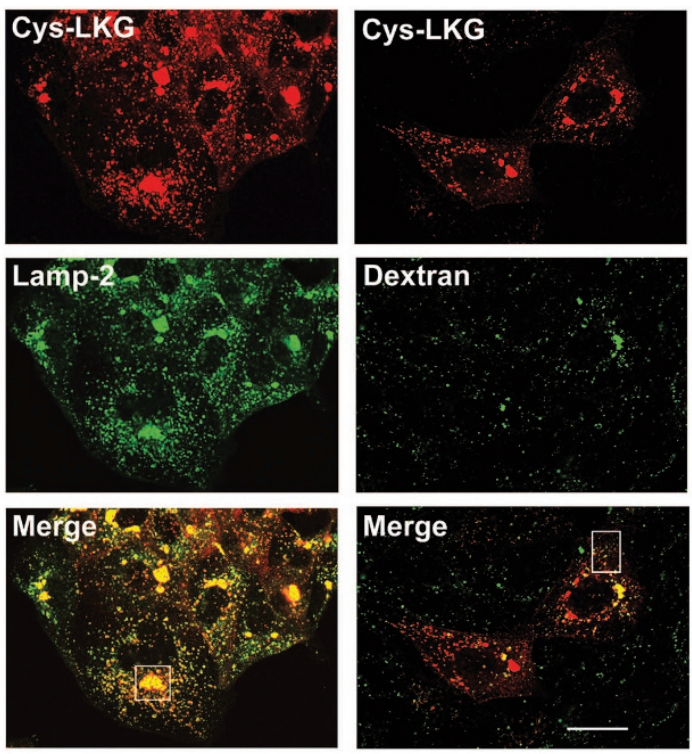

C

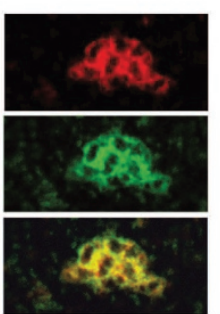

d

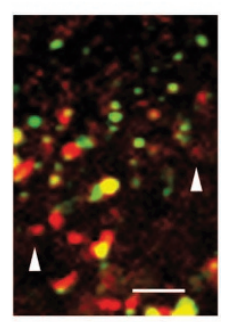

Figure 1. Lysosomal localization of cystinosin-LKG. (a) Confocal microscopy images of MDCK II cells expressing cystinosin-RFP (Cys) and cystinosinLKG-RFP (Cys-LKG) stained with anti-Lamp2 antibody or loaded in vivo with alexa 488-dextran. (b) The colocalization degree of cys-RFP (light gray) and cys-LKG-RFP (dark gray) with Lamp2 was $95.2 \pm 3.4$ and $73.1 \pm 10.8 \%$ (mean $\pm S D$ ), ${ }^{*} P<0.001$, respectively. Data are representative of at least 30 cells. (c) High-magnification images of boxed area showing enlarge perinuclear structure representing cys-LKG-RFP (red), Lamp2 (green), and their overlapping signals (yellow). (d) High-magnification image of boxed area representing cys-LKG-RFP and dextran colocalization. Dextran-negative vesicles (white arrowheads). Scale bars: $30 \mu \mathrm{m}$ in panel a and $20 \mu \mathrm{m}$ in panel d.

pancreatic islets of Langerhans, Leydig cells of the testis, mucoserous glands of the bronchial wall, melanocytes, and keratinocytes (15).

In this study, we have further investigated cystinosin-LKG distribution in renal epithelial cells and analyzed its capacity to rescue the cystinotic phenotype.

\section{RESULTS}

\section{Cystinosin-LKG in Lysosomes}

We first studied the subcellular localization of the cystinosinLKG in MDCK II cells overexpressing cystinosin-LKG conjugated with the red fluorescence protein DsRed (cysLKG-RFP). Similarly to cystinosin (cys-RFP), cys-LKG-RFP extensively localized in Lamp2-positive vesicles, some of which appeared as large perinuclear structures (Figure 1a,c). The same distribution was observed after loading cells in vivo with alexa-488 dextran (Figure 1a, third column). As shown in Figure 1b, both cys-LKG-RFP $(73.1 \pm 10.8 \%)$ and cys-RFP $(95.2 \pm 3.4 \%)$ colocalized with Lamp2; however, the degree of colocalization was stronger for cys-RFP $(P<0.001)$. Colocalization analyses and high-magnification images revealed that cys-LKG-RFP was also localized in
Lamp2-negative vesicles (Figure 1d, white arrowheads). To further characterize perinuclear structures, MDCK II cells expressing cys-RFP or cys-LKG-RFP were labeled with antibody directed against Lamp2 and processed for immuneelectron microscopy studies. Lamp2-positive structures (arrows) were distributed throughout the cell cytoplasm and had an usual lysosomal morphology in control MDCK II cells (Figure 2a). In contrast, MDCK II cells expressing cys-RFP showed several perinuclear Lamp2-positive multivesicular bodies (Figure 2b). Lamp2-positive perinuclear structures of MDCK II cells expressing cys-LKG-RFP were characterized by the accumulation of stacked lamellar membranes (Figure 2c).

\section{Cystinosin-LKG in Intracellular Organelles}

The expression of cystinosin-LKG in Lamp2-negative structures has prompted to further investigate its localization in other vesicular compartments. MDCK II cells expressing cysLKG-RFP were incubated with antibodies binding markers for endoplasmic reticulum (PDI), cis-Golgi apparatus (gm130), early endosomes (EEA1), late endosomes (Alix), and peroxisomes (Catalase). 

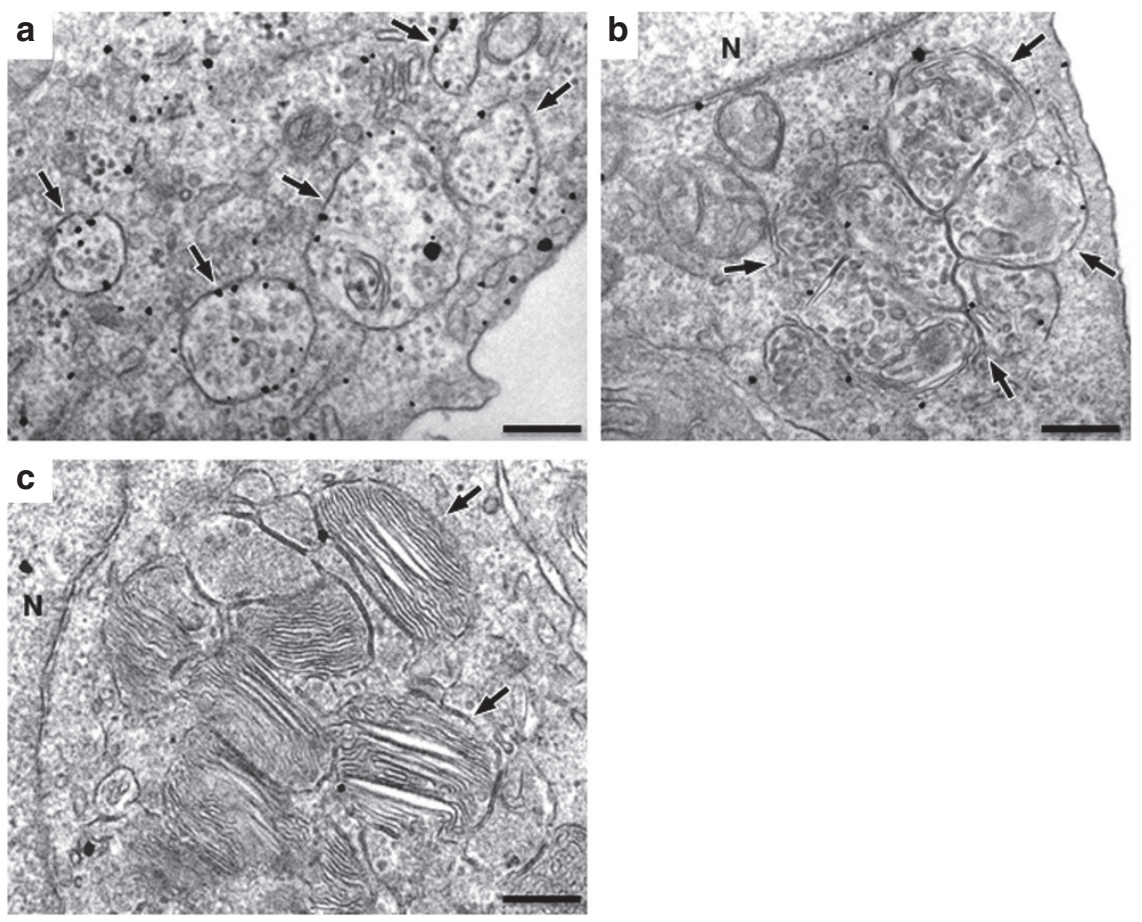

Figure 2. Ultrastructural analysis of lysosomes. Electron microscopy images of MDCK II cells (a) expressing cystinosin-RFP (b) or cystinosin-LKG-RFP (c) processed for immunocytochemistry (immunogold) with anti-Lamp2 antibody (lysosomal marker) and revealed with secondary antibody conjugated to gold particles. Perinuclear Lamp-2 positive multivesicular bodies and parallel lamellar vesicules (arrows) were observed in cells expressing cystinosin and cystinosin-LKG, respectively. N: Nucleus. Scale bar: $200 \mathrm{~nm}$.

As shown in Figure 3, cystinosin-LKG colocalized in part with Alix $(40.89 \pm 1.81 \%)$ and PDI $(27.87 \pm 1.64 \%)$, and was only occasionally observed in structures marked with EEA1 $(10.93 \pm 0.58 \%)$, gm130 $(8.4 \pm 0.63 \%)$, and catalase $(7.40 \pm 0.61 \%)$.

\section{Cystinosin-LKG in the Plasma Membrane of Polarized MDCK II Cells}

In order to study the expression of the cystinosin-LKG in polarized cells, MDCK II cells expressing cys-LKG-RFP (Figure 4a,d) were grown on polycarbonate filters for $96 \mathrm{~h}$, fixed and stained for E-cadherin (Figure 4b) and gp135 (Figure 4e), two proteins that are located on the baso-lateral and apical surfaces, respectively. Cystinosin-LKG overlapped with gp135 protein on the apical surface (Figure 4f), whereas fluorescence signal at the baso-lateral aspects did not colocalize with E-cadherin (Figure 4c).

\section{Cystinosin-LKG Decreases Lysosomal Cystine Accumulation in Cystinotic Proximal Tubular Epithelial Cells}

To determinate whether cystinosin-LKG is available to mediate the cystine efflux in lysosomes, cys-LKG-RFP or cysRFP (positive control) or RFP (negative control) were stably expressed in immortalized human proximal tubular epithelial cells (PTEC) carrying the $57 \mathrm{~kb}$ deletion of the Ctns gene (cystinotic PTEC). Cystine levels were significantly higher $(3.53 \pm 0.63 \mathrm{nmol} / \mathrm{mg}$ of protein) in cystinotic PTEC compared to wild-type cells $(0.54 \pm 0.35 \mathrm{nmol} / \mathrm{mg}$ of protein). The expression of RFP did not modify cystine accumulation in cystinotic PTEC (Figure 5a). Cystine levels were significantly decreased from $3.24 \pm 0.14 \mathrm{nmol} / \mathrm{mg}$ of protein in cystinotic cells to $0.42 \pm 0.014 \mathrm{nmol} / \mathrm{mg}$ of protein $(P<0.005)$ and $0.32 \pm 0.015 \mathrm{nmol} / \mathrm{mg}$ of protein $(P<0.005)$ in cystinotic cells after expressing cystinosin-LKG or cystinosin, respectively (Figure 5a). Both isoforms restored cystine levels to levels similar to those observed in wild-type PTEC (Figure 5a).

\section{Cystinosin-LKG Preserves Cystinotic PTEC From Apoptosis}

Because cystinotic cells are more sensitive to apoptotic stimuli than healthy cells, the protective role of cystinosinLKG against apoptosis was investigated in cystinotic PTEC. Apoptotis was induced by $0.03 \mu \mathrm{g} / \mathrm{ml} \mathrm{TNF} \alpha$ and $2.5 \mu \mathrm{g} / \mathrm{ml}$ actinomycin D for $16 \mathrm{~h}$ in wild-type and cystinotic PTEC. The number of annexin $\mathrm{V}$-positive cells was significantly higher in cystinotic PTEC under basal conditions $(6.69 \pm 1.78$-folds, $P<0.01)$ and after stimulation with TNF $\alpha /$ actinomycin D (21.5 \pm 4.82 -folds, $P<0.005)$, compared to control wildtype cells $(1 \pm 0.13$, Figure 5 b). Based on these results, we transiently transfected cystinotic cells with vectors carrying cDNA for RFP, cystinosin-RFP, or cystinosin-LKG-RFP, to compare the rate of apoptosis between transfected and untrasfected cells; nontransfected cells in the same culture dish were used as internal controls. The rate of apoptosis relative to untransfected cystinotic cells of the same cellular culture, dropped to $0.62 \pm 0.01(P<0.005)$ and $0.48 \pm 0.01$ $(P<0.005)$ in cystinotic PTEC expressing cystinosin-LKG or cystinosin, respectively (Figure $5 \mathrm{c}$ ). This effect was not observed in cells transfect with RFP (Figure 5c). 


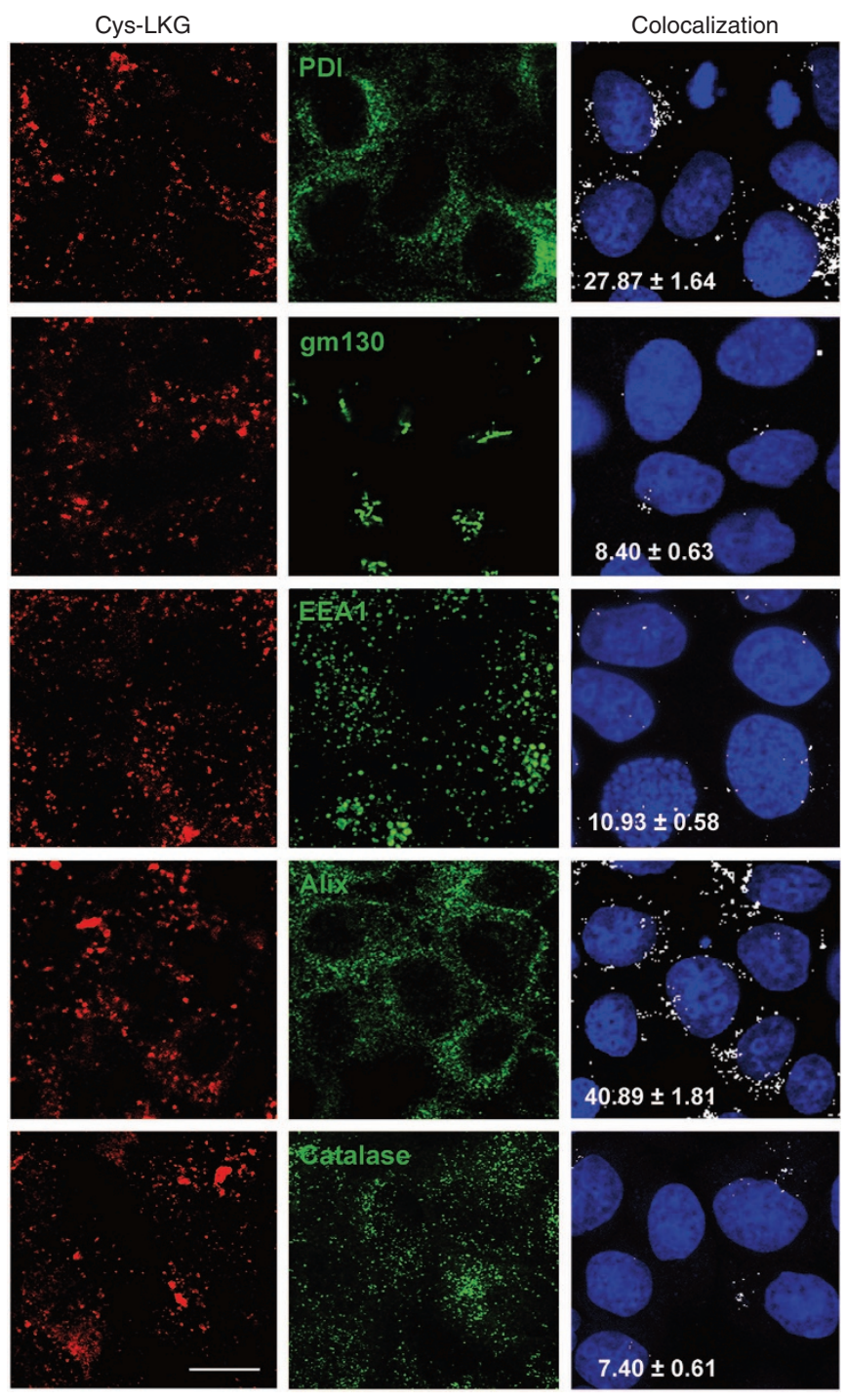

Figure 3. Subcellular distribution of cystinosin-LKG. Confocal microscopy images of MDCK II cells expressing cystinosin-LKG-RFP (Cys-LKG) stained with antibodies for endoplasmic reticulum (anti-PDI), cis-Golgi apparatus (anti-gm130), early endosomes (anti-EEA1), late endosomes (anti-alix) and peroxisomes (anti-catalase). The nuclei were stained with Hoechst (blue). Colocalization masks (visualized as white spots) exhibited the overlap pattern between two markers. Numbers indicate the percentage of colocalization (mean \pm SEM) that was valued on at least 45 cells for each marker. Scale bar: $20 \mu \mathrm{m}$.

\section{DISCUSSION}

After having identified the cystinosin-LKG, the finding that its intracellular localization is not restricted to the lysosomal compartments has raised questions on the role of this isoform in tissues, in particular in proximal tubular epithelial cell $(13,15)$. Alternative splicing in higher eukaryotic organisms enables a single gene to produce different proteins (16). Solute carrier genes by alternative splicing strategies encode for distinct transmembrane transporters that differ in their tissue distribution, subcellular localization, and physiological proprieties, allowing the development of sophisticated transport systems (17). For examples in the field of the renal transporters, the slc14a2 gene encodes five isoforms of urea transporter
(UT-A) that differ in the carboxyl or amino terminal domains, and cause differences in the phosphorylation sensitivity after vasopressin stimulation and in tissue distribution (18). UT-A 1-4 isoforms have been described in the inner medullary collection duct or in the thin descending limb, whereas the UT-A 5 isoform has been found exclusively in the testis. Similarity the $s l c 12 a 1$ gene encodes three $\mathrm{Na}-\mathrm{K}-\mathrm{Cl}$ cotransporter isoforms with diverse carboxyl-terminal sequences (NKCC2). These isoforms show different localizations along the thick ascending limb of the loop of Henle and affinity for $\mathrm{NaCl}$ (19).

The ctns gene primary transcript is spliced in several mRNA transcripts, including cystinosin-LKG. The cystinosin of Drosophila melanogaster (CG17119) is 397 amino acid long, lacks a "GYDQL" amino acid sequence in the carboxyl-terminal tail and is more similar to cystinosin-LKG. This isoform could therefore represent an ancetral cystine transporter that subsequently evolved into cystinosin. However, it is remarkable that cystinosin-LKG has been preserved throughout evolution, raising the possibility of specific functions related to this isoform. We have studied the capacity of cystinosin-LKG to rescue the cystinotic phenotype in epithelial cells by investigating two of the main cellular defects that have been reported in renal cystinotic cells.

Both studied isoforms had the same capacity of rescuing cystine accumulation and apoptosis, although they have differences in their subcellular localization. In addition, we have recently demonstrated that the carboxyl-terminal sequence of cystinosin-LKG plays an important role in plasma membrane sorting (20) Taken together, these data suggest a different role of cystinosin-LKG in vesicular traffic, which is currently been evaluated in our laboratory.

DMT1 gene (divalent metal transport), through alternative splicing, encodes two isoforms that exhibit different carboxylterminal tails, that confer specific vesicular distribution and iron binding (21).

Abnormal lysosomal morphology has been described in various lysosomal storage diseases that are caused by dysfunctional amino acid efflux or impaired substrate digestion (22). Enlarged lysosomes for example, have been described in Caenorhabditis elegant qx42 mutant lacking the lysosomal lysine/arginine transporter LAAT-1, causing lysosomal amino acid accumulation (23). The unexpected formation of zebra like structures in MDCK II cells expressing cys-LKG-RFP, could be due to lysosomal engulfment caused by high expression of transporter in the lysosomes. This hypothesis is suggested by the observation of similar parallel lamellar bodies in Hurler and Niemann-Pick diseases that are characterized by gangliosides and cholesterol lysosomal accumulation, respectively (24) (www.pathologyoutlines.com). Alternatively, this finding could be related to enhanced autophagy.

Our in vitro studies have shown expression of cys-LKG-RFP on the apical membrane in polarized MDCK II cells. As has been previously observed, cystinosin-LKG seems to be concentrated in the basal aspects of the renal proximal tubules and gastric mucosa epithelial cells, in the apical aspects of ciliated pseudostratified bronchial epithelium cells, and around the 

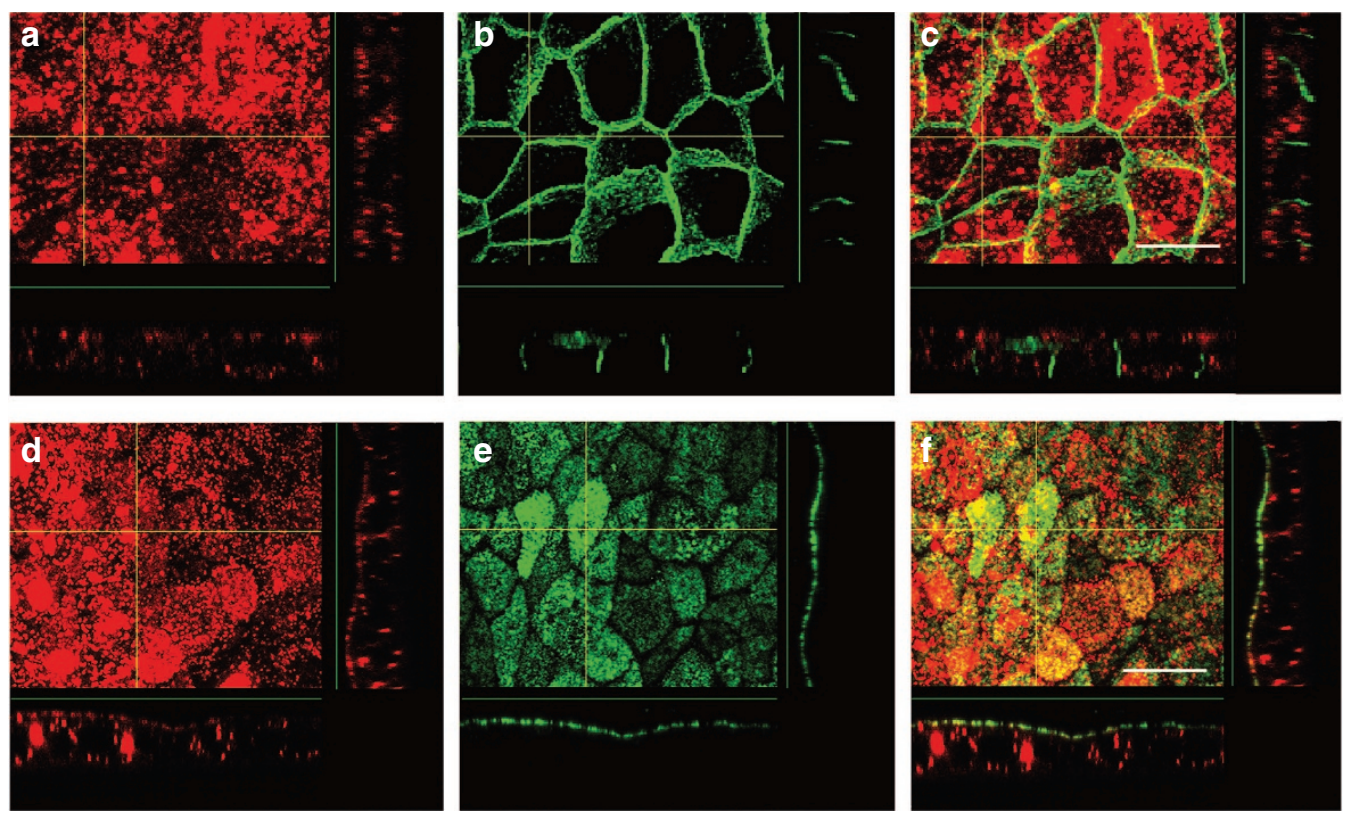

Figure 4. Localization of cystinosin-LKG in polarized cells. Z-reconstructions obtained by confocal microscopy of MDCK II cells expressing cystinosin-LKG$\operatorname{RFP}(\mathbf{a}, \mathbf{d})$ polarized on polycarbonate filters and stained with antibodies against E-cadherin (b) and gp135 (e). Distributions of cys-LKG-RFP/E-cadherin (c) in baso-lateral faces (upper panel) and cys-LKG-RFP/gp135 (f) in the apical face (lower panel). Merged images are shown in the right column. Scale bars: $10 \mu \mathrm{m}$ in the upper panel, $20 \mu \mathrm{m}$ in the lower panel.

nuclei in Langerhans islet cells. As for other transporters, this suggest cell-specific distribution of the protein in tissues (25).

In conclusion, this study has improved our understanding of the physiological role of the cystinosin-LKG. It remains to be clarified if cystinosin-LKG can substitute the more abundant cystinosin isoform. This question is extremely important in the light of possible gene therapy for nephropathic cystinosis. Our results show that both isoforms have very similar functions. However, since both isoforms have been preserved throughout evolution, there are probably subtle differences that we still do not fully understand.

\section{METHODS}

Cloning of Cystinosin-LKG cDNA Conjugated With DsRed-Coding Sequence Into pcDNA3.1 Vector

pDsRed-N1 plasmid carrying human cystinosin-LKG cDNA (CTNS-LKG; accession number: NM_001031681) conjugated with red fluorescence protein (RFP) coding sequence was used as donor of CTNS-LKG-RFP (13). After digestion with NheI (New England Biolabs, Ipswich, MA) and NotI (Thermo Scientific, Fermentas, Waltham, MA) restriction enzymes, CTNS-LKG-RFP was cloned into pcDNA3.1 plasmid, using T4 DNA Ligase (Thermo Scientific) in according to the manufacturer's protocol. Analogue procedure was performed for cloning human cystinosin cDNA (CTNS; accession number: NM_004937) conjugated with RFP into pcDNA3.1 (CTNS-RFP).

\section{Stable or Transient Transfection in MDCK II and PTEC}

pcDNA3.1 plasmid carrying CTNS-LKG-RFP was transfected with Lipofectamine 2000 LXT with plus (Life Technologies, Invitrogen, Paisley, UK) into MDCK II or cystinotic PTEC cells. For stable transfection, MDCK II cells expressing cystinosin-LKG-RFP (cysLKG-RFP) were selected with $200 \mathrm{\mu g} / \mathrm{ml}$ hygromycin B for $14 \mathrm{~d}$ (Life Technologies), whereas PTEC expressing cys-LKG-RFP were isolated by cell sorting (FACSAria II; BD Biosciences, San Jose, CA). Analogue procedure was performed for cells expressing cystinosin-RFP (cysRFP). MDCK II cells were cultured in Dulbecco's modified essential medium (Gibco, Grand Island, NY), supplemented with $10 \%$ fetal calf serum (Gibco), $2 \mathrm{mmol} / \mathrm{l} \mathrm{L-glutamine,} 100$ units $/ \mathrm{ml}$ penicillin and 100 $\mu \mathrm{g} / \mathrm{ml}$ streptomycin. PTEC were cultured in Dulbecco's modified essential medium/F12 (Gibco), supplemented with $10 \%$ fetal calf serum (Gibco), ITS ( $5 \mu \mathrm{g} / \mathrm{ml}$ insulin, $5 \mu \mathrm{g} / \mathrm{ml}$ transferrin, $5 \mathrm{ng} / \mathrm{ml}$ selenium), $36 \mathrm{ng} / \mathrm{ml}$ hydrocortisone, $10 \mathrm{ng} / \mathrm{ml}$ epidermal growth factor, $40 \mathrm{pg} / \mathrm{ml}$ triiodothyronine, $100 \mathrm{units} / \mathrm{ml}$ penicillin, and $100 \mu \mathrm{g} / \mathrm{ml}$ streptomycin. These last reagents were supplied by Sigma Aldrich (Milan, Italy).

Cystinotic PTEC were kindly provided by Elena Levtchenko (Department of Pediatrics, University Hospitals Leuven, Belgium). PTEC were isolated from urine of cystinotic and healthy donors, infected with SV40T and hTERT vectors, and characterized with antibodies against epithelial markers (26).

\section{Immunofluorescence Assay}

Cells expressing cys-LKG-RFP were fixed with $4 \%$ paraformaldehyde for $10 \mathrm{~min}$ at $4{ }^{\circ} \mathrm{C}$, permeabilized with $0.05 \%$ Triton $\mathrm{X}-100$ for $10 \mathrm{~min}$ at $4{ }^{\circ} \mathrm{C}$, incubated with $5 \%$ bovine serum albumin in phosphate-buffered saline and 5\% normal goat serum (NGS) for $30 \mathrm{~min}$ at room temperature (RT). Cells were incubated with primary monoclonal antibodies at following conditions: Lamp2 (dilution 1:10, overnight at $4{ }^{\circ} \mathrm{C}$; Santa Cruz Biotechnology, Santa Cruz, CA), Lamp1 (dilution 1:10, overnight at $4{ }^{\circ} \mathrm{C}$; Santa Cruz Biotechnology), PDI (protein disulphide-isomerase, dilution 1:500, $60 \mathrm{~min}$ at RT; Life Technologies), EEA1 (dilution 1:200, 60 min at RT; BD Transduction Laboratories, San Jose, CA), gm130 (dilution 1:200, 60 min at RT; BD Transduction Laboratories), Alix (dilution $1: 10$, overnight at $4{ }^{\circ} \mathrm{C}$; Santa Cruz Biotechnology), catalase (dilution 1:100, overnight at $4{ }^{\circ} \mathrm{C}$; Abcam, Cambridge, UK). Then, samples were incubated with Alexa Fluor 488 goat anti-mouse antibody (Life Technologies). For nuclear staining, $1 \mu \mathrm{g} / \mathrm{ml}$ of Hoechst (Life Technologies) was used.

To load lysosomes in vivo, MDCK II cells were incubated in culture medium containing $0.5 \mathrm{ng} / \mathrm{ml}$ Alexa Fluor 488 dextran (Molecular Probes, Waltham, MA) at $37^{\circ} \mathrm{C}$, overnight, washed twice with culture medium and followed by chasing in medium lacking dextran for $2 \mathrm{~h}$.

\section{Polarization of MDCK II Cells}

MDCK cells expressing cys-LKG-RFP were seeded at a density of $4 \times 10^{5}$ cells $/ \mathrm{cm}^{2}$ on Transwell polycarbonate filters, $12 \mathrm{~mm}$ diameter, $0.4 \mu \mathrm{m}$ pore size (Corning Costar, Lowell, MA) and cultured for 7 d. Cells were fixed with $4 \%$ paraformaldehyde for $10 \mathrm{~min}$ at $4{ }^{\circ} \mathrm{C}$, 
a

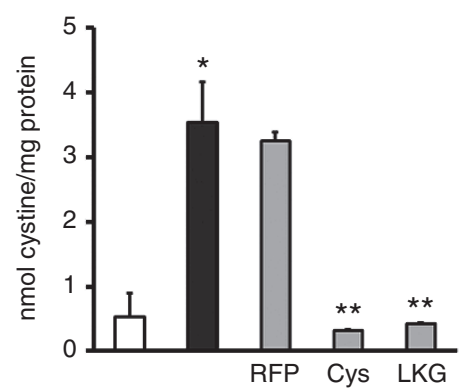

b

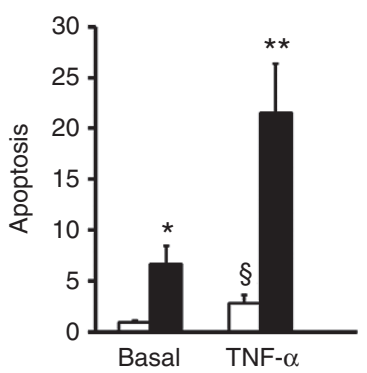

C

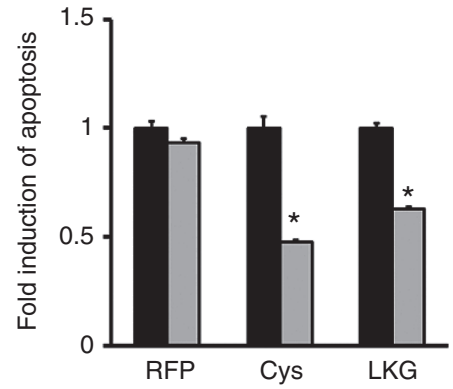

Figure 5. Function of cystinosin-LKG in cystinotic proximal tubular epithelial cells (PTEC). (a) Cystine levels were analyzed by HPLC in wild-type (white column) and cystinotic PTEC (black column) expressing RFP, cystinosinRFP (Cys) or cystinosin-LKG-RFP (LKG) (grey columns). Cystine levels were higher in cystinotic PTEC respect to wild-type PTEC $\left(n=4,{ }^{*} P<0.005\right)$ and decreased in cystinotic PTEC expressing cystinosin or cystinosin-LKG ( $n$ $=3,{ }^{*} P<0.005$ vs. cystinotic PTEC). Data are expressed as means \pm SD. Statistical comparison was determined by student's two-tailed $t$-test. (b) The rate of apoptosis in wild-type (white columns) and cystinotic (black columns) PTEC stimulated with TNF $\alpha$ and actinomycin D. Cells were stained with annexin V-FITC and measured by flow cytometry. The rate of apoptosis was increased in cystinotic PTEC at basal condition $\left({ }^{*} P<0.01\right)$ and under apoptotic stimuli $(* *<0.005)$, and in wild-type PTEC under apoptotic stimuli ( $\left.{ }^{5} P<0.007\right)$ compared to wild-type cells at basal condition. Data are expressed as means \pm SEM of three independent experiments. (c) The fold induction of apoptosis in cystinotic cells (black columns) expressing RFP, cystinosin and cystinosin-LKG (grey columns). Both isoforms decreased the fold induction of apoptosis after stimulation with TNF $\alpha$ and actinomycin $D$ $\left({ }^{*} P<0.005\right.$ vs. untransfected cystinotic PTEC). Data are expressed as means \pm SEM of three independent experiments.

permeabilized with $0.2 \%$ Triton X-100 for $15 \mathrm{~min}$ at $4{ }^{\circ} \mathrm{C}$, blocked nonspecific sites with 5\% BSA/PBS and 5\% NGS for $30 \mathrm{~min}$ at RT, incubated with rat anti-E-cadherin (Sigma Aldrich) or mouse antigp135 antibodies (kindly provided by George Ojakian, Department of Cell Biology SUNY Downstate Medical Center, Brooklin, NY) for $2 \mathrm{~h}$ at RT, then revealed with Alexa Fluor 488-conjugated secondary antibodies (Life Technologies).

\section{Cystine Measurement}

For cystine measurements, the cells were sonicated in the presence of $10 \mathrm{mmol} / \mathrm{l} \mathrm{N}$-ethylmaleimide. The protein fraction was precipitated by the addition of $10 \% 5$-sulfosalicylic acid and measured using the Pierce bicinchoninic acid protein assay. Cystine levels were measured by highperformance liquid chromatography, as previously described (27).

\section{Apoptosis Assay}

After apoptotic stimuli, cells were detached by trypsin $(0.5 \times)$ and suspended in complete culture medium for PTEC. Rapid annexin V binding protocol was chosen between methods suggested by Calbiochem annexin V-FITC apoptosis detection kit (Merck Millipore, Darmstadt, Germany). Briefly, media binding reagent and annexin V-FITC were added to cell suspension and incubated for $15 \mathrm{~min}$, in the dark. Cells were collected by centrifugation, suspended in binding buffer, and analyzed by flow cytometry (FACSCanto II, BD Biosciences).

\section{Confocal Microscopy}

Images were acquired using an Olympus FV1000 laser-scanning confocal microscope (FV10-ASW software) using the $63 \times$ oil objective (numerical aperture 1.4). Optical single sections were acquired with a scanning mode format of $1,024 \times 1,024$ pixels, with a pixel size of $0.21 \mu \mathrm{m}$ sampling speed of $40 \mu \mathrm{s} /$ pixel and $12 \mathrm{bits} /$ pixel images. Z-reconstructions of serial single optical sections were carried out with an electronic zoom at 2 and $Z$ stack of $0.5 \mu \mathrm{s} /$ slice. Fluorochromes unmixing was performed by acquisition of automated-sequential collection of multi-channel images, in order to reduce spectral crosstalk between channels. The threshold-based approach was performed to obtain colocalization masks using the colocalization function provided by FV10-ASW Olympus.

Confocal and reconstructed images were assembled in Adobe Photoshop software (Adobe Systems, CA).

\section{Electron Microscopy}

MDCK cells stably expressing cys-LKG-RFP were fixed with a mixture of $4 \%$ paraformaldehyde and $0.05 \%$ glutaraldehyde, permeabilized and labeled with an antibody against Lamp2. Then samples were dehydrated in ethanol and acetone and embedded in epoxy resin as described previously (28). From each sample, thin $65 \mathrm{~nm}$ sections were cut using a Leica EM UC7 ultramicrotome. EM images were acquired from thin sections using a FEI Tecnai-12 electron microscope (FEI, Eindhoven, Netherlands) equipped with a VELETTA CCD digital camera (Soft Imaging Systems GmbH, Munster, Germany).

\section{STATEMENT OF FINANCIAL SUPPORT}

This study was supported by grant of the Cystinosis Research Foundation (Irvine, CA).

\section{Disclosure: There is no conflict of interest to disclose.}

\section{REFERENCES}

1. Town M, Jean G, Cherqui S, et al. A novel gene encoding an integral membrane protein is mutated in nephropathic cystinosis. Nat Genet 1998;18:319-24.

2. Gahl WA, Thoene JG, Schneider JA. Cystinosis. N Engl J Med 2002;347: 111-21.

3. Wilmer MJ, Emma F, Levtchenko EN. The pathogenesis of cystinosis: mechanisms beyond cystine accumulation. Am J Physiol Renal Physiol 2010;299:F905-16.

4. Johnson JL, Napolitano G, Monfregola J, Rocca CJ, Cherqui S, Catz SD. Upregulation of the Rab27a-dependent trafficking and secretory mechanisms improves lysosomal transport, alleviates endoplasmic reticulum stress, and reduces lysosome overload in cystinosis. Mol Cell Biol 2013;33:2950-62.

5. Raggi C, Luciani A, Nevo N, Antignac C, Terryn S, Devuyst O. Dedifferentiation and aberrations of the endolysosomal compartment characterize the early stage of nephropathic cystinosis. Hum Mol Genet 2014;23: 2266-78.

6. Sansanwal P, Yen B, Gahl WA, et al. Mitochondrial autophagy promotes cellular injury in nephropathic cystinosis. J Am Soc Nephrol 2010;21: 272-83.

7. Bellomo F, Corallini S, Pastore A, et al. Modulation of CTNS gene expression by intracellular thiols. Free Radic Biol Med 2010;48:865-72.

8. Gaide Chevronnay HP, Janssens V, Van Der Smissen P, et al. Time course of pathogenic and adaptation mechanisms in cystinotic mouse kidneys. J Am Soc Nephrol 2014;25:1256-69.

9. Park M, Helip-Wooley A, Thoene J. Lysosomal cystine storage augments apoptosis in cultured human fibroblasts and renal tubular epithelial cells. J Am Soc Nephrol 2002;13:2878-87.

10. Park MA, Pejovic V, Kerisit KG, Junius S, Thoene JG. Increased apoptosis in cystinotic fibroblasts and renal proximal tubule epithelial cells results from cysteinylation of protein kinase Cdelta. J Am Soc Nephrol 2006;17:3167-75.

11. Sansanwal P, Li L, Sarwal MM. Inhibition of intracellular clusterin attenuates cell death in nephropathic cystinosis. J Am Soc Nephrol 2015;26: 612-25. 
12. Cherqui S, Kalatzis V, Trugnan G, Antignac C. The targeting of cystinosin to the lysosomal membrane requires a tyrosine-based signal and a novel sorting motif. J Biol Chem 2001;276:13314-21.

13. Taranta A, Petrini S, Palma A, et al. Identification and subcellular localization of a new cystinosin isoform. Am J Physiol Renal Physiol 2008;294:F1101-8.

14. Kalatzis V, Nevo N, Cherqui S, Gasnier B, Antignac C. Molecular pathogenesis of cystinosis: effect of CTNS mutations on the transport activity and subcellular localization of cystinosin. Hum Mol Genet 2004;13:1361-71.

15. Taranta A, Petrini S, Citti A, et al. Distribution of cystinosin-LKG in human tissues. Histochem Cell Biol 2012;138:351-63.

16. Blencowe BJ. Alternative splicing: new insights from global analyses. Cell 2006;126:37-47.

17. Hediger MA, Romero MF, Peng JB, Rolfs A, Takanaga H, Bruford EA. The ABCs of solute carriers: physiological, pathological and therapeutic implications of human membrane transport proteinsIntroduction. Pflugers Arch 2004;447:465-8.

18. Sands JM. Molecular mechanisms of urea transport. J Membr Biol 2003;191:149-63.

19. Castrop H, Schnermann J. Isoforms of renal $\mathrm{Na}-\mathrm{K}-2 \mathrm{Cl}$ cotransporter NKCC2: expression and functional significance. Am J Physiol Renal Physiol 2008;295:F859-66.

20. Bellomo F, Taranta A, Petrini S, et al. Carboxyl-terminal SSLKG motif of the human cystinosin-LKG plays an important role in plasma membrane sorting. PLoS One 2016;11:e0154805.
21. Tabuchi M, Tanaka N, Nishida-Kitayama J, Ohno H, Kishi F. Alternative splicing regulates the subcellular localization of divalent metal transporter 1 isoforms. Mol Biol Cell 2002;13:4371-87.

22. Parkinson-Lawrence EJ, Shandala T, Prodoehl M, Plew R, Borlace GN, Brooks DA. Lysosomal storage disease: revealing lysosomal function and physiology. Physiology (Bethesda) 2010;25:102-15.

23. Liu B, Du H, Rutkowski R, Gartner A, Wang X. LAAT-1 is the lysosomal lysine/arginine transporter that maintains amino acid homeostasis. Science 2012;337:351-4.

24. Perkins DG, Haust MD. Ultrastructure of myocardium in the Hurler syndrome. Possible relation to cardiac function. Virchows Arch A Pathol Anat Histol 1982;394:195-205.

25. Devuyst O, Christie PT, Courtoy PJ, Beauwens R, Thakker RV. Intra-renal and subcellular distribution of the human chloride channel, CLC-5, reveals a pathophysiological basis for Dent's disease. Hum Mol Genet 1999;8: 247-57.

26. Wilmer MJ, Kluijtmans LA, van der Velden TJ, et al. Cysteamine restores glutathione redox status in cultured cystinotic proximal tubular epithelial cells. Biochim Biophys Acta 2011;1812:643-51.

27. Pastore A, Lo Russo A, Greco M, Rizzoni G, Federici G. Semiautomated method for determination of cystine concentration in polymorphonuclear leukocytes. Clin Chem 2000;46:574-6.

28. Polishchuk EV, Concilli M, Iacobacci S, et al. Wilson disease protein ATP7B utilizes lysosomal exocytosis to maintain copper homeostasis. Dev Cell 2014;29:686-700. 